\title{
Qualidade nutricional da polpa de bocaiúva Acrocomia aculeata (Jacq.) Lodd.
}

\author{
Nutritional quality of the pulp of bocaiuva Acrocomia aculeata (Jacq.) Lodd. \\ Maria Isabel Lima RAMOS ${ }^{1 \star}$, Manoel Mendes RAMOS FILHO ${ }^{1}$, Priscila Aiko HIANE ${ }^{1}$, \\ José Antonio BRAGA NETO ${ }^{1}$, Egle Machado de Almeida SIQUEIRA ${ }^{2}$
}

\section{Resumo}

Com o objetivo de incentivar o consumo e o aproveitamento de alimentos oriundos do Cerrado Brasileiro, foram determinados os teores de umidade, lipídios totais, proteínas, carboidratos, cinzas, fibra, minerais e os principais carotenóides da polpa de bocaiúva, Acrocomia aculeata (Jacq.) Lodd. A porção comestível do fruto (polpa e amêndoa) representou aproximadamente a metade do peso total do fruto. A polpa apresentou $52,99 \%$ de umidade, $8,14 \%$ de lipídios totais, $1,5 \%$ de proteínas, $22,08 \%$ de carboidratos, $1,51 \%$ de cinzas e $13,76 \%$ de fibra. O valor energético foi estimado em $167,67 \mathrm{kcal} .100 \mathrm{~g}^{-1}$ de polpa úmida, o qual mostrou-se superior ao de outros frutos da

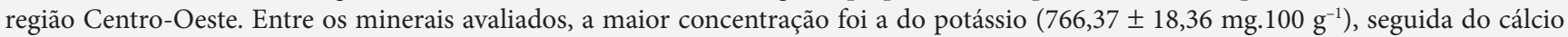
$\left(61,96 \pm 2,30 \mathrm{mg} .100 \mathrm{~g}^{-1}\right)$ e do fósforo $\left(36,70 \mathrm{mg} \cdot 100 \mathrm{~g}^{-1}\right)$. Relacionando-se os resultados de minerais às IDR de referências dos mesmos, a polpa de bocaiúva pode ser classificada como rica em cobre para crianças, como fonte de zinco e potássio para crianças e como fonte de cobre e potássio para adultos. A polpa mostrou-se rica em $\beta$-caroteno $\left(49,0 \pm 2,0 \mu \mathrm{g} \cdot \mathrm{g}^{-1}\right.$ de polpa integral), principal carotenóide identificado no fruto analisado, podendo contribuir com o enriquecimento da dieta regional em programas de suplementação alimentar, como uma fonte natural desse nutriente e dos minerais cobre, potássio e zinco.

Palavras-chave: frutos do cerrado; bocaiúva; nutrientes; minerais; carotenóides.

\begin{abstract}
With the aim at stimulating the consumption of natural food from the Brazilian Cerrado (vast tropical savanna ecoregion of Brazil), the contens of moisture, lipid, protein, carbohydrate, ash, fiber, minerals, and main carotenoids were determinated in the bocaiuva Acrocomia aculeata (Jacq.) Lodd. pulp. The edible portion of the fruit (pulp and kernel) corresponded to $50 \%$ of the whole fruit. The pulp presented $52.99 \%$ of moisture, $8.14 \%$ of total lipids, $1.5 \%$ of protein, $22.08 \%$ of carbohydrates, $1.51 \%$ of ash, and $13.76 \%$ of fiber. The energetic value was estimated to be $167.67 \mathrm{kcal} .100 \mathrm{~g} \mathrm{~g}^{-1}$ of wet pulp, which is higher than the other fruits from this region. Among the minerals examined, potassium was the element in higher concentrations $\left(766.37 \pm 18.36 \mathrm{mg} .100 \mathrm{~g} \mathrm{~g}^{-1}\right)$ followed by calcium $\left(61.96 \pm 2.30 \mathrm{mg} .100 \mathrm{~g}^{-1}\right)$ and copper $\left(36.70 \pm 0.0 \mathrm{mg} .100 \mathrm{~g}^{-1}\right)$. The bocaiuva pulp may be classified as a rich source of copper for children, as a source of zinc and potassium for children, and as a source of copper and potassium for adults according to the dietary recommendation intake of those minerals. The bocaiuva pulp can be considered as rich in $\beta$-carotene $\left(49.0 \pm 2.0 \mu \mathrm{g} . \mathrm{g}^{-1}\right.$ of integral pulp), the predominant carotenoid identified in the pulp, which can contribute to the enrichment of the regional diet, in supplementary diet programs, as a natural source of that nutrient as well as of vitamin A, copper, potassium and zinc minerals. Keywords: native fruits; bocaiuva; nutrients; minerals; carotenoids.
\end{abstract}

\section{Introdução}

$\mathrm{Na}$ região Centro-Oeste, onde predomina o cerrado, ocorrem muitas espécies de frutas nativas consideradas fontes de proteínas, fibras, energia, vitaminas, cálcio, fósforo e ácidos graxos (SILVA et al., 1994; SANO; ALMEIDA, 1998; MARIN, 2006). Muitas frutas são comercializadas e consumidas in natura ou processadas em preparo caseiro, com grande aceitação popular (ALMEIDA, 1998). O aproveitamento tecnológico de espécies frutíferas nativas pode constituir uma preciosa fonte de alimentos e riqueza para o país.

A bocaiúva é um fruto que pertence à família Palmae, sendo a espécie Acrocomia aculeata (Jacq.) Lodd. abundante e nativa no Estado do Mato Grosso do Sul. A polpa e a amêndoa na forma in natura são consumidas pela população local ou nas preparações da culinária regional, tais como sorvetes, bolos, paçoca doce e cocada, podendo enriquecer a dieta como fonte complementar de nutrientes essenciais (ALMEIDA, 1998; SANO; ALMEIDA, 1998).

Frutas e vegetais são exemplos de importantes fontes de nutrientes essenciais, entre eles, encontram-se os minerais, que desempenham uma função vital no desenvolvimento e boa saúde do corpo humano. Os minerais são essenciais à manutenção de várias funções de importância fisiológica como na contratibilidade muscular, na função dos nervos, na coagulação sanguínea, nos processos digestivos e no equilíbrio ácido-básico (FRANCO, 2004; HARDISSON et al., 2001).

Hortaliças e frutas também são consideradas fontes de carotenóides, os quais atuam não apenas como corantes naturais, mas como compostos bioativos com atuação benéfica à

Recebido para publicação em 26/4/2007

Aceito para publicação em 8/5/2008 (002490)

${ }^{1}$ Departamento de Tecnologia de Alimentos e Saúde Pública, Universidade Federal de Mato Grosso do Sul - UFMS, CEP 79070-900, Campo Grande - MS, Brasil,

E-mail:milramos@nin.ufms.br

${ }^{2}$ Departamento de Biologia Celular, Universidade de Brasília - UnB, CEP 70910-900, Brasília - DF, Brasil

${ }^{*}$ A quem a correspondência deve ser enviada 
saúde humana. A principal atividade é atribuída à capacidade de conversão em vitamina A, que no organismo está relacionada à visão, ao crescimento ósseo e à diferenciação de tecidos (OLSON, 1999; IOM, 2001). Atuam também na redução de risco de patologias como câncer, doenças cardiovasculares, cataratas, desordens fotossensíveis e do sistema imunológico (SOMMER, 1995; PAHO, 2001; TAPIERO, TAWNSEND; TEW, 2004). A composição em carotenóides nesses alimentos pode ser afetada por vários fatores, entre eles a variedade, cultivar, estágio de maturação, condições climáticas e geográficas de produção, manuseio durante e pós-colheita, processamento e estocagem (RODRIGUEZ-AMAYA, 1999).

Frutos do cerrado e do pantanal têm sido avaliados quanto à composição em nutrientes e carotenóides, visando o conhecimento de matérias-primas regionais (RAMOS et al., 2001; HIANE et al., 2003; CALDEIRA et al., 2004).

Este estudo teve como objetivo avaliar a composição centesimal, os minerais e os principais carotenóides da polpa de bocaiúva Acrocomia aculeata (Jacq.) Lodd., contribuindo para o aproveitamento tecnológico e nutricional de frutos da região.

\section{Material e métodos}

\subsection{Matéria-prima}

Frutos maduros de bocaiúva, A. aculeata (Jacq.) Lodd., foram coletados no solo do campus da Universidade Federal de Mato Grosso do Sul e em algumas regiões próximas a Campo Grande, MS, entre os meses de agosto e novembro de 2005. Todos os frutos foram reunidos e posteriormente selecionados, procurando-se obter um lote uniforme quanto ao tamanho e ao grau de maturação, que foi determinado pela intensidade de cor (amarelo intenso). Deste lote, separaram-se 20 frutos aleatoriamente para a avaliação das características físicas, medindo-se o diâmetro, o peso do fruto, da casca (epicarpo), da polpa (mesocarpo), da semente composta de casca dura (endocarpo) e da amêndoa. O restante dos frutos (cerca de 300 unidades) foi descascado, despolpado manualmente e a polpa homogeneizada em processador de alimentos, embalada e armazenada $-20^{\circ} \mathrm{C}$ até o início das análises.

\subsection{Composição centesimal}

A polpa homogeneizada foi avaliada quanto à composição centesimal, de acordo com os métodos descritos nas normas analíticas do Instituto Adolfo Lutz (BRASIL, 2005) e Association of Official Analytical Chemists - AOAC (1992).

A determinação de umidade foi feita por dessecação em estufa a $105{ }^{\circ} \mathrm{C}$ (método gravimétrico), o teor de lipídios totais determinado pelo método de extração direta com solvente orgânico em aparelho de Soxhlet, as cinzas (resíduo mineral fixo) foram determinadas por calcinação em mufla a $550^{\circ} \mathrm{C}$ (método gravimétrico), e os carboidratos avaliados através do método de Lane-Eynon, de acordo com metodologias descritas nas normas analíticas do Instituto Adolfo Lutz (BRASIL, 2005). A proteína foi determinada pelo conteúdo de nitrogênio total (\%), segundo método microKjeldahl, usando-se o fator 6,25 para a conversão do nitrogênio em proteínas, descrito na AOAC (1992). A fibra foi obtida por diferença entre $100 \mathrm{~g}$ e as gramas totais de umidade, proteína, lipídios, cinzas e carboidratos “disponíveis” (USP, 2005).

A energia total proveniente dos nutrientes foi expressa em quilocalorias (kcal), estimada a partir dos fatores de conversão de Atwater: $\mathrm{kcal}=(4 \times \mathrm{g}$ proteína $)+(4 \times \mathrm{g}$ carboidratos $)+$ (9 x g lipídios) (USP, 2005).

\subsection{Teores de minerais}

Os teores de minerais foram avaliados em amostras trituradas e homogeneizadas, preparadas de acordo com metodologia descrita por Salinas e Garcia (1985), com digestão orgânica por via úmida. Na digestão orgânica, as amostras foram tratadas com uma mistura de ácido nítrico e ácido perclórico, ambos concentrados, em alta temperatura, e os macro e microelementos presentes foram solubilizados, submetidos a diferentes tratamentos e diluídos para posterior avaliação quantitativa. A quantificação dos elementos foi realizada por espectrofotometria, utilizando-se de curva padrão para cada mineral. Para determinação da concentração de cálcio, ferro, manganês, zinco e cobre, empregou-se espectrofotômetro de absorção atômica (Perkim-Elmer mod. 2380) e gás acetileno. Utilizou-se fotômetro de chama (Micronal B262) para determinação de sódio (589 nm) e potássio $(768 \mathrm{~nm})$ e espectrofômetro luz visível (Femto 482) para a determinação de fósforo (420 nm).

\subsection{Composição em carotenóides}

A polpa do fruto homogeneizada (25 g) foi submetida à extração com acetona resfriada, os carotenóides transferidos para o éter de petróleo e o extrato saponificado com solução metanólica de hidróxido de potássio a 30\%, durante uma noite, em temperatura ambiente, de acordo com as metodologias descritas por Rodriguez-Amaya (1999) e Hiane e Penteado (1989). Todas as etapas foram desenvolvidas, utilizando proteção com tecido preto e/ou papel alumínio, evitando a foto degradação dos carotenóides.

O extrato etéreo contendo os carotenóides foi concentrado em evaporador rotatório (temperatura inferior a $40^{\circ} \mathrm{C}$ ) e o resíduo obtido foi submetido à análise através de cromatografia líquida de alta eficiência (CLAE). Utilizou-se equipamento HPLC (Waters modelo 2695) com detector UV-Visível e arranjo de diodos (Waters modelo 2996), coluna monomérica C18 Spherisorb OD2 (3 $\mu \mathrm{m}, 4,6 \times 150 \mathrm{~mm})$, fase móvel acetonitrila (0,05\% de trietilamina):metanol:acetato de etila, com gradiente linear de 90:10:0 a 60:20:20 em 40 minutos, fluxo de $0,5 \mathrm{~mL} /$ minuto e detecção a $450 \mathrm{~nm}$. A quantificação do $\beta$-caroteno foi realizada através do método de padronização externa (KIMURA; RODRIGUEZ-AMAYA, 2002). A polpa homogeneizada foi analisada em triplicata.

\section{Resultados e discussão}

\subsection{Características físicas}

A porção comestível composta pela polpa e amêndoa, representou $48 \%$ do peso total do fruto (Tabela 1 ), mostrando 
bom rendimento para preparações culinárias, aproveitamento tecnológico e para o fornecimento de elementos potencialmente nutritivos. Os frutos apresentaram diâmetro médio de $3,38 \pm 0,15 \mathrm{~cm}$, semelhante ao encontrado em frutos oriundos de Minas Gerais, Brasil, descritos por Almeida (1998) $(3 \mathrm{~cm}$ ), porém mostraram diferentes proporções de casca (epicarpo), polpa (mesocarpo) e semente (endocarpo e amêndoa).

\subsection{Composição centesimal}

A polpa de bocaiúva mostrou-se rica em lipídios totais, carboidratos e fibra (Tabela 2). Comparando a composição da bocaiúva encontrada neste estudo com a obtida anteriormente por Hiane et al. (1992), observa-se que o teor de umidade para a polpa estudada foi superior a $49,14 \%$, porém, o teor de lipídios foi aproximadamente a metade do percentual $(16,50 \%)$ e, conseqüentemente, apresentou menor valor calórico, provavelmente em função do tipo de solo, condições climáticas, período de coleta e outros fatores que afetam a composição dos frutos.

A polpa de bocaiúva analisada apresentou potencial energético duas vezes superior ao de outra palmeira da região, o buriti $\left(85,9 \mathrm{kcal} .100 \mathrm{~g}^{-1}\right.$ de polpa integral) e cerca de três vezes superior ao de outros frutos regionais (caraguatá, pitanga e araticum, araçá e tarumã), cujos valores energéticos apresentaram variação de 39 a $55 \mathrm{kcal}^{1} 100 \mathrm{~g}^{-1}$ de polpa integral (HIANE et al., 1992; CALDEIRA et al., 2004). Os teores de lipídios e carboidratos encontrados na polpa representaram cerca de 40 e $50 \%$ da energia total oriunda dos macronutrientes.

Tabela 1. Características físicas do fruto, Acrocomia aculeata (Jacq.) Lodd.

\begin{tabular}{lcc}
\hline \multicolumn{1}{c}{ Medidas físicas } & Massa $(\mathrm{g}) \pm \mathrm{DP}^{*}$ & $\%$ \\
\hline Fruto inteiro & $21,83 \pm 2,48$ & \multicolumn{1}{c}{$\%$} \\
Casca (epicarpo) & $4,68 \pm 1,07$ & 21,44 \\
Polpa (mesocarpo) & $9,61 \pm 1,17$ & 44,22 \\
Semente (endocarpo e amêndoa) & $7,55 \pm 1,23$ & 34,58 \\
Amêndoa & $0,83 \pm 0,23$ & 3,80 \\
\hline
\end{tabular}

${ }^{*}$ Resultado médio e desvio padrão de 20 frutos.

Tabela 2. Composição centesimal da polpa de bocaiúva, Acrocomia aculeata (Jacq.) Lodd., expressa em g. $100 \mathrm{~g}^{-1}$ de amostra úmida.

\begin{tabular}{lr}
\hline \multicolumn{1}{c}{ Componentes } & Média $\pm \mathrm{DP}^{*}$ \\
\hline Umidade $(\% \mathrm{~m} / \mathrm{m})$ & $52,99 \pm 2,88$ \\
Resíduo mineral fixo $(\% \mathrm{~m} / \mathrm{m})$ & $1,51 \pm 0,06$ \\
Lipídios totais $(\% \mathrm{~m} / \mathrm{m})$ & $8,14 \pm 1,45$ \\
Proteínas $(\% \mathrm{~m} / \mathrm{m})$ & $1,50 \pm 0,04$ \\
Glicose $(\% \mathrm{~m} / \mathrm{m})$ & $9,47 \pm 2,48$ \\
Sacarose $(\% \mathrm{~m} / \mathrm{m})$ & $0,07 \pm 0,17$ \\
Amido $(\% \mathrm{~m} / \mathrm{m})$ & $12,56 \pm 0,48$ \\
Fibra por diferença & $13,76 \pm 4,07$ \\
Valor calórico total $\left(\mathrm{kcal} .100 \mathrm{~g}^{-1}\right)$ & $167,67 \pm 2,56$ \\
\hline
\end{tabular}

*Valores médios de 3 determinações \pm desvio padrão.

\subsection{Teores de minerais}

Dentre os elementos minerais analisados, o que apresentou maior concentração foi o potássio, seguido do cálcio e do fósforo (Tabela 3). Comparando os teores de minerais da polpa com os da amêndoa obtidos por Hiane et al. (2006), observou-se que o teor do potássio foi duas vezes superior ao da amêndoa (377,2 mg.100 g $\mathrm{g}^{-1}$ de amostra integral), porém os elementos fósforo, sódio, ferro, manganês, zinco e cobre encontrados na polpa, mostraram-se inferiores. Geralmente, são esperados teores de minerais em amêndoas superiores aos de polpas, pois as amêndoas apresentam menores conteúdos de umidade e seus elementos nutritivos mais concentrados.

Comparando-se os teores de minerais da bocaiúva com os encontrados em partes comestíveis de frutas tropicais comercializadas e consumidas pela população (abacate, abacaxi, banana, mamão, maracujá, melão e tangerina) (GONDIM, 2005), observa-se que os teores de cálcio e potássio foram superiores aos de todas essas frutas, ressaltando que o teor de potássio foi o dobro do encontrado na banana $\left(333,4 \mathrm{mg} \cdot 100 \mathrm{~g}^{-1}\right)$ e no maracujá (380,0 mg.100 g $\left.\mathrm{g}^{-1}\right)$, os quais são considerados importantes fontes desse mineral (FRANCO, 2004).

Os teores de minerais encontrados em $100 \mathrm{~g}$ de polpa de bocaiúva não suprem a cota dietética mínima recomendada para adultos (BRASIL, 2005; NATIONAL, 2006). Porém, comparando esses teores com a IDR de referência do mineral e a classificação de alimento como rico ou fonte de um mineral quando proporciona 30 ou $15 \%$ da ingestão diária recomendável/100 g de polpa, respectivamente, de acordo com Portaria no 31/1998 - SVS/MS (1998), a polpa de bocaiúva pode ser classificada como rica em cobre para crianças e como fonte para adultos de qualquer idade, proporcionando 71 e $27 \%$ da IDR de referência, respectivamente. Os conteúdos dos elementos zinco e potássio proporcionaram 20 e $25 \%$ da IDR de referência para crianças (1-3 anos), respectivamente, e 16\% da IDR do potássio para adultos, classificando a polpa como fonte desses nutrientes.

\subsection{Composição em carotenóides}

O cromatograma do extrato de carotenóides da polpa de bocaiúva obtido através da técnica de CLAE apresentou 10 picos, os quais foram identificados por meio do detector

Tabela 3. Teores de minerais da polpa de bocaiúva, Acrocomia aculeata (Jacq.) Lodd., expressos em amostra úmida.

\begin{tabular}{lc}
\hline Mineral & $\mathrm{mg} \cdot 100 \mathrm{~g}^{-1}$ \\
\hline Cálcio & $61,96 \pm 2,30$ \\
Fósforo & $36,70 \pm 0,00$ \\
Potássio & $766,37 \pm 18,36$ \\
\hline & $\mu \mathrm{g} \cdot \mathrm{g}^{-1 \star}$ \\
\hline Sódio & $3,74 \pm 0,25$ \\
Ferro & $7,81 \pm 0,22$ \\
Manganês & $1,38 \pm 0,00$ \\
Zinco & $6,02 \pm 0,11$ \\
Cobre & $2,43 \pm 0,00$ \\
\hline
\end{tabular}

${ }^{*}$ Média e desvio padrão de duas repetições. 
de arranjo de diodos como sendo: 1-zeaxantina, 4-translicopeno, 5- $\alpha$-criptoxantina ou zeinoxantina, 6-cis-licopeno, 7 e 8 - $\gamma$-caroteno, 9 -trans $\beta$-caroteno e 10 -13-cis- $\beta$-caroteno (Figura 1).

O teor de $\beta$-caroteno encontrado na polpa da bocaiúva foi de 49,0 $\mu \mathrm{g} \cdot \mathrm{g}^{-1}$ de polpa úmida de $\beta$-caroteno total, correspondendo a cerca de $80 \%$ dos carotenóides totais encontrados na polpa (pico 9, Figura 1). Os demais carotenóides minoritários identificados nas alíquotas analisadas não foram quantificados neste estudo. O conteúdo de $\beta$-caroteno foi próximo ao valor encontrado por Hiane e Penteado em polpa de bocaiúva analisada através de cromatografia em coluna aberta $\left(59,41 \pm 11,09 \mu \mathrm{g} . \mathrm{g}^{-1}\right)$, porém diferindo em sua composição, pois foram identificados os carotenóides $\beta$-caroteno, $\gamma$-caroteno, $\beta$-criptoxantina, cislicopeno e cis-flavoxantina, com predominância do $\beta$-caroteno (89\%), em relação aos carotenóides totais presentes na polpa (HIANE; PENTEADO, 1989). O teor de $\beta$-caroteno da polpa de bocaiúva foi superior ao encontrado em outros frutos do cerrado do pantanal sul-mato-grossense. Para as polpas comestíveis de pindó (Arecastrum romanozoffianum Becc.), bacuri (Scheelea phalerata Mart.), piqui (Caryocar brasiliense Camb.) e caraguatá (Bromelia balansae Mez) foram detectados 36,6; 17,3; 14,7 e $0,5 \mu \mathrm{g} . \mathrm{g}^{-1}$ de amostra úmida de $\beta$-caroteno, respectivamente (ALONSO et al., 1998; ANDREOLLA et al., 1998; RAMOS et al., 2001; HIANE et al., 2003). Segundo Rodriguez-Amaya (1999), outras palmeiras frutíferas brasileiras também foram estudadas, entre elas, o buriti (Mauritia flexuosa, L.), o tucumã (Astrocarium vulgare) e a pupunha (Bactris gasipaes), as quais foram consideradas ricas em carotenóides, com predominância de $\beta$-caroteno, $\alpha$-caroteno e $\gamma$-caroteno.

Embora as novas recomendações do Institute of Medicine (IOM) adotem fatores de conversão do $\beta$-caroteno e dos carotenóides duas vezes maiores que os fatores utilizados no passado (IOM, 2001; CAMPOS; ROSADO, 2005), e dificultem a proposta de atingir as recomendações dessa vitamina com o consumo de vegetais e frutos ricos em carotenóides pró-vitamínicos $\mathrm{A}$, estudos recentes têm demonstrado que vegetais, frutas e leguminosas podem suprir quantidades significativas de vitamina A em animais e humanos (TANG et al., 2005; DOSTIL et al., 2006; SIQUEIRA et al., 2007).

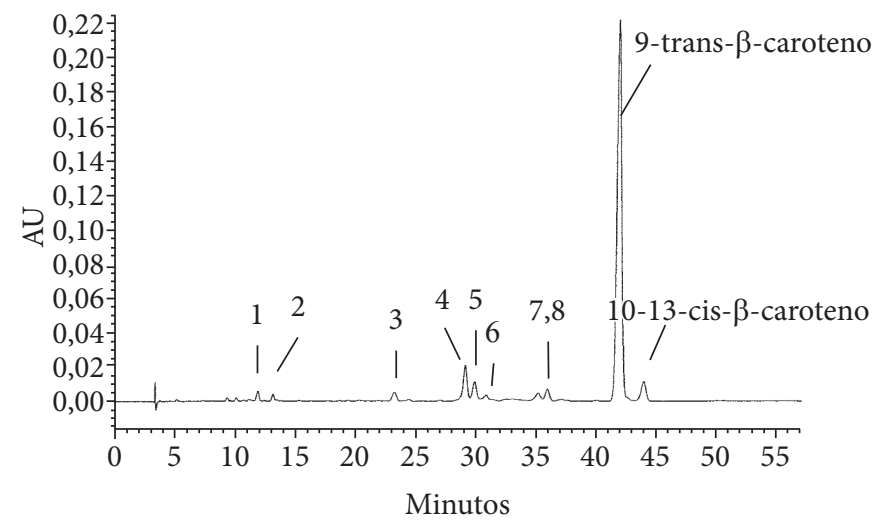

Figura 1. Cromatograma obtido por HPLC.
$\mathrm{Na}$ avaliação do potencial nutritivo do alimento, deve-se considerar não somente a concentração dos nutrientes presentes no alimento, mas também a biodisponibilidade dos nutrientes deste alimento. A biodisponibilidade dos carotenóides alimentares é afetada por muitos fatores endógenos e exógenos (VAN HET HOF et al., 2000; YEUM; RUSSEL, 2002; DOSTIL et al., 2006). Assim, estudos adicionais sobre a biodisponibilidade do $\beta$-caroteno devem ser incentivados visando avaliar a real contribuição de frutos como fonte de vitamina A. Um estudo recente revelou que o $\beta$-caroteno da polpa da bocaiúva é altamente biodisponível em relação ao $\beta$-caroteno puro (RAMOS et al., 2007). Estes resultados evidenciam o potencial da polpa de bocaiúva como alimento nutritivo, capaz de contribuir para o enriquecimento da dieta regional em programas de suplementação alimentar, como uma fonte natural de $\beta$-caroteno e de vitamina A.

\section{Conclusões}

A porção comestível do fruto bocaiúva, A. aculeata (Jacq.) Lodd., constituída de polpa e amêndoa mostrou bom rendimento para o aproveitamento tecnológico e fornecimento de elementos potencialmente nutritivos.

Os teores de minerais encontrados indicaram que a polpa de bocaiúva pode ser classificada como rica em cobre para crianças e como fonte de zinco e potássio para elas, além de ser fonte de cobre e potássio para adultos.

A polpa de bocaiúva mostrou-se rica em $\beta$-caroteno, podendo contribuir com o enriquecimento da dieta regional em programas de suplementação alimentar, como uma fonte natural desse nutriente e de vitamina $\mathrm{A}$, bem como dos minerais cobre, potássio e zinco.

\section{Agradecimentos}

Agradecemos à Doutora Mieko Kimura, Ibilce/Universidade Estadual de São Paulo, pelo apoio nas análises de carotenóides no fruto.

\section{Referências bibliográficas}

ALMEIDA, S. P. Cerrado: aproveitamento alimentar. Planaltina, DF: EMBRAPA, Centro de Pesquisa Agropecuária dos Cerrados - CPAC, $1998.188 \mathrm{p}$.

ALONSO, C. P. et al. Carotenóides do pindó (Arecastrum romanozoffianum Becc.). Identificação e quantificação de próvitamínicos A. In: CONGRESSO BRASILEIRO DE CIÊNCIA E TECNOLOGIA DE ALIMENTOS,16, 1998, Rio de Janeiro. Anais... Rio de Janeiro: SBCTA, 1998.

ANDREOLLA, A. et al. Carotenóides pró-vitamínicos A do caraguatá (Bromelia balansae Mez) nativo do Estado de Mato Grosso do Sul. In: CONGRESSO BRASILEIRO DE CIÊNCIA E TECNOLOGIA DE ALIMENTOS, 16, 1998, Rio de Janeiro. Anais... Rio de Janeiro: SBCTA, 1998.

ASSOCIATION OF OFFICIAL ANALYTICAL CHEMISTS. Official methods of analysis of AOAC International. $12 \mathrm{ed}$. Washington, 1992. $1115 \mathrm{p}$. 
BRASIL. Ministério da Saúde. Agência Nacional de Vigilância Sanitária (ANVISA). Métodos Físico-Químicos para Análise de Alimentos. Brasília: Ministério da Saúde, 2005. 1018 p.

BRASIL. Ministério da Saúde. Agência Nacional de Vigilância Sanitária (ANVISA). Resolução RDC n. 269, de 22 de setembro de 2005. Regulamento técnico sobre a ingestão diária recomendada (IDR) de proteína, vitaminas e minerais. Diário Oficial [da] República Federativa do Brasil, Brasília, 23 de setembro de 2005.

BRASIL. Ministério da Saúde. Secretaria de Vigilância Sanitária. Portaria n. 31, de 13 de janeiro de 1998. Regulamento técnico para fixação de identidade e qualidade de alimentos adicionados de nutrientes essenciais. Diário Oficial [da] República Federativa do Brasil, Brasília, 16 de janeiro de 1998.

CALDEIRA, S. D. et al. Caracterização físico-química do araçá (Psidium guineense SW.) e do tarumã (Vitex cymosa Bert.) do Estado de Mato Grosso do Sul. Boletim do Centro de Pesquisa e Processamento de Alimentos, v. 22, n. 1, p. 145-154, 2004.

CAMPOS, F. M.; ROSADO, G. P. Novos fatores de conversão de carotenóides provitamínicos A. Ciência e Tecnologia de Alimentos, v. 25 , n. 3, p. $571-578,2005$.

DOSTI, M. P. et al. Bioavailability of $\beta$-carotene $(\beta C)$ from purple carrots is the same as typical orange carrots while high- $C$ carrots increase $\beta \mathrm{C}$ stores in Mongolian gerbils (Meriones unguiculatus). British Journal of Nutrition, v. 96, n. 2, p. 258-267, 2006.

FRANCO, G. Tabela de composição química dos alimentos. 9 ed. São Paulo: Editora Atheneu, 2004. 307 p.

GONDIM, J. A. M. et al. Composição centesimal e de minerais em cascas de frutas. Ciência Tecnologia de Alimentos, v. 25, n. 4, p. 825-827, 2005.

HARDISSON, A. et al. Mineral composition of the banana (Musa acuminata) from the island Tenerife. Food Chemistry, v. 17, n. 2, p. 153-161, 2001.

HIANE, P. A. et al. Chemical and nutrition evaluation of kernels of bocaiuva, Acrocomia aculeata (Jacq.) Lodd. Ciência e Tecnologia de Alimentos, v. 26, n. 3, p. 683-689, 2006.

HIANE, P. A. et al. Carotenóides pró-vitamínicos A e composição em ácidos graxos do fruto e da farinha de bacuri (Scheelea phalerata Mart.). Ciência e Tecnologia de Alimentos, v. 23, n. 2, p. 206-209, 2003.

HIANE, P. A.; PENTEADO, M. V. C. Carotenóides e valor de vitamina A do fruto e da farinha de bocaiúva (Acrocomia mokayáyba Barb. Rodr.) do Estado de Mato Grosso do Sul. Revista de FarmáciaBioquímica da Universidade de São Paulo, v. 25, n. 2, p. 158-168, 1989.

HIANE, P. A. et al. Composição centesimal e perfil de ácidos graxos de alguns frutos nativos do Estado de Mato Grosso do Sul. Boletim do Centro de Pesquisa e Processamento de Alimentos, v. 10, n. 1, p. 35-42, 1992.

IOM - U.S. INSTITUTE OF MEDICINE. Food and Nutrition Board, Standing Committee on the Scientific Evaluation of Dietary Reference Intakes. Dietary Reference Intakes: for Vitamin A, Vitamin K, Arsenic, Boron, Cromium, Copper, Iodine, Iron, Manganese, Molybdenium, Nickel, Silicon, Vanadium and Zinc. Washington, D. C.: National Academy Press, 2001. 797 p.

KIMURA, M.; RODRIGUEZ-AMAYA, D. B. A scheme for obtaining standards and HPLC quantification of leafy vegetable carotenoids. Food Chemistry, v. 78, n. 3, p. 389-398, 2002.
MARIN, A. M. F. Potencial nutritivo de frutos do Cerrado: composição em minerais e componentes não convencionais. Brasília, 2006. 121 p. Dissertação - (Mestrado em Nutrição), Departamento de Nutrição, Universidade de Brasília - UnB.

NATIONAL ACADEMIES PRESS. Dietary reference intakes: elements. 2002. Disponível em: <http://www.nal.usda.gov>. Acesso em: 21 fev. 2006.

OLSON, J. A. Bioavailability of carotenoids. Archivos Latinoamericanos de Nutricion, v. 49, Suppl 1, p. 26S-33S, 1999.

PAHO - Pan American Health Organization. Providing vitamin A supplements through immunization and other health contacts for children 0-59 months and women up to 6 weeks postpartum: A guide for health workers. 2 ed. Washington, 2001. 35 p.

RAMOS, M. I. L. et al. Efeito do cozimento convencional sobre os teores de carotenóides pró-vitamínicos A da polpa do piqui (Caryocar brasiliense Camb.). Boletim do Centro de Pesquisa e Processamento de Alimentos, v. 19, n. 1, p. 23-32, 2001.

RAMOS, M. I. L. et al. Bocaiuva (Acrocomia aculeata (Jacq) Lodd) improved Vitamin A status in rats. Journal of Agricultural and Food Chemistry, v. 22, n. 8, p. 3186-3190, 2007.

RODRIGUEZ-AMAYA, D. B. A guide to carotenoid analysis in foods. Washington: OMNI Research, 1999. 64 p.

RODRIGUEZ-AMAYA, D. B. Latin American food sources of carotenoids. Archivos Latinoamericanos de Nutrición, n. 49, Suppl 1, p. 74S-84S, 1999.

SALINAS, Y. G.; GARCIA, R. Métodos químicos para el análisis de suelos acidos y plantas forrajeras. Cali: Centro Internacional de Agricultura Tropical, 1985. 83 p.

SANO, S. M.; ALMEIDA, S. P. (Ed.). Cerrado-ambiente e flora. Planaltina: EMBRAPA - Centro de Pesquisa Agropecuária dos Cerrados - CPAC, 1998. 556 p.

SILVA, J. A. et al. Frutas nativas dos cerrados. Brasília, DF: EMBRAPA, Centro de Pesquisa Agropecuária dos Cerrados - CPAC, 1994. $166 \mathrm{p}$.

SIQUEIRA, E. M. A. et al. $\beta$-Carotene from cassava (Manihot esculenta Crantz) leaves improves vitamin A status in rats. Comparative Biochemistry and Physiology, C, Toxicology and Pharmacology, v. 146, n. 1-2, p. 235-240, 2007.

SOMMER, A. La carencia de vitamina A y sus consecuencias. Guia práctica para la detección y el tratamiento. 3 ed. Genebra: Organización Mundial de la Salud, 1995.

TANG, G. et al. Spinach or carrots can supply significant amounts of vitamin A as assessed by feeding with intrinsically deuterated vegetables. American Journal of Clinical Nutrition, v. 82, n. 4, p. 821-828, 2005.

TAPIERO, H.; TOWNSEND, D. M.; TEW, K. D. The role of carotenoids in the prevention of human pathologies. Biomedicine and Pharmacotherapy, v. 58, n. 2, p. 100-110, 2004.

USP. Universidade de São Paulo. Tabela de composição de alimentos: projeto integrado de composição de alimentos. Disponível em: <http:/www.fcf.usp.br/tabela/>. Acesso em: 28 dez 2005.

VAN HET HOF, K. H. et al. Carotenoid bioavailability in humans from tomatoes processed in different ways from the carotenoid response in the triglyceride-rich lipoprotein fraction of plasma after a single consumption and in plasma after four days of consumption. Journal of Nutrition, v. 130, n. 5, p. 1189-1196, 2000.

YEUM, K. L.; RUSSEL, R. M. Carotenoid bioavailability and conversion. Annual Review of Nutrition, v. 22, jul, p. 483-504, 2002. 